\title{
REAL-TIME ESTIMATION OF PROJECTILE ROLL ANGLE USING MAGNETOMETERS: IN-LAB EXPERIMENTAL VALIDATION
}

\author{
S. Changey, E. Pecheur, P. Wey, and E. Sommer \\ French-German Research Institute of Saint-Louis (ISL) \\ P.O. Box 70034, Saint-Louis 68301, France
}

\begin{abstract}
The knowledge of the roll angle of a projectile is decisive to apply guidance and control law. For example, the goal of ISL's project GSP (Guided Supersonic Projectile) is to change the flight path of an airdefence projectile in order to correct the aim error due to the target manoeuvres. The originality of the concept is based on pyrotechnical actuators and onboard sensors which control the angular motion of the projectile. First of all, the control of the actuators requires the precise control of the roll angle of the projectile. To estimate the roll angle of the projectile, two magnetometers are embedded in the projectile to measure the projection of the Earth magnetic field along radial axes of the projectiles. Then, an extended Kalman filter (EKF) is used to compute the roll angle estimation. As the rolling frequency of the GSP is about $22 \mathrm{~Hz}$, it was easy to test the navigation algorithm in laboratory. In a previous paper [1], the In-Lab demonstration of this concept showed that the roll angle estimation was possible with an accuracy of about $1^{\circ}$. In this paper, the demonstration is extended to high-speed roll rate, up to $1000 \mathrm{~Hz}$. Thus, two magnetometers, a DSP (Digital Signal Processor) and a LED (Light Eminent Diode), are rotated using a pneumatic motor; the DSP runs an EKF and a guidance algorithm to compute the trigger times of the LED. By using a high-speed camera, the accuracy of the method can be observed and improved.
\end{abstract}

\section{GUIDED SUPERSONIC PROJECTILE}

For ground-based air defence gun systems to be effective, the ability to hit the target is a prerequisite. To achieve high hit probability performance against manoeuvring air targets, such as attack helicopters, cruise missiles, or unmanned air vehicles, the basic point is to fire projectiles having short times of flight. However, this solution is limited by the performance of the gun. This paper 


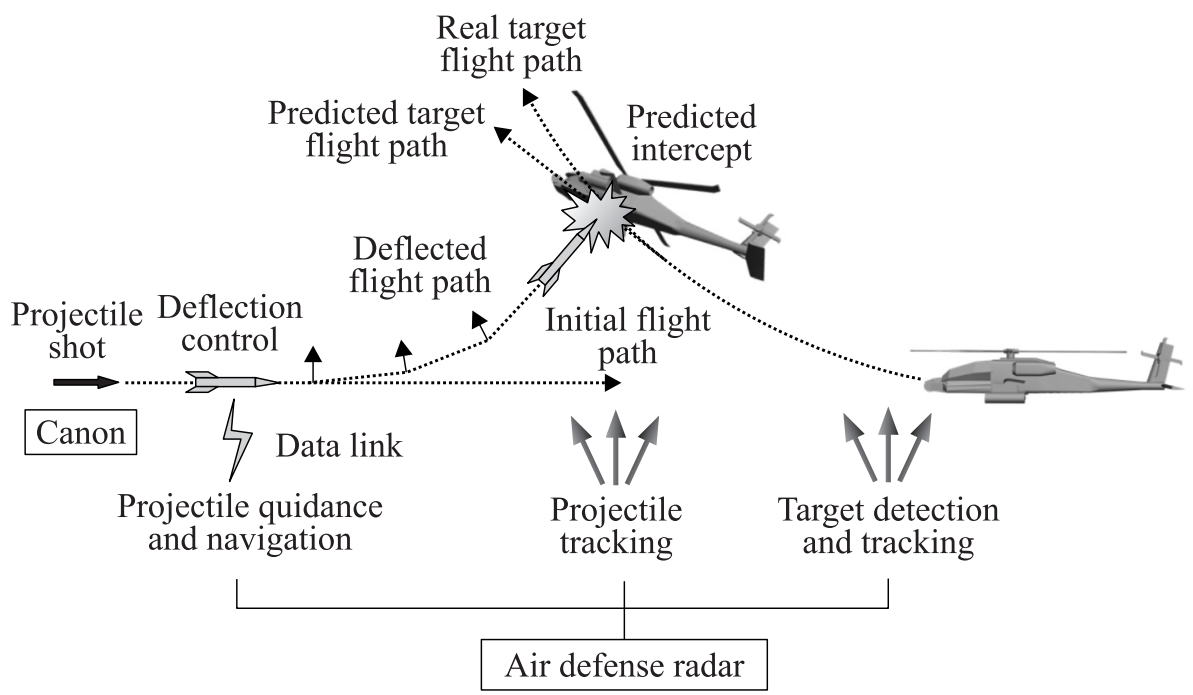

Figure 1 Overview of the GSP concept

describes an alternative approach based on guided projectiles with embedded maneuver capabilities.

Figure 1 shows a block diagram of the GSP concept designed by ISL [2]. It consists of a supersonic 30-millimeter finned projectile with onboard navigation sensors and lateral impulse thrusters. The system is designed for very short range air defence $(<5 \mathrm{~km})$.

The initial target tracking and fire control processes are supposed to be performed by standard systems. The target is tracked using a battlefield-type air defence radar. The initial flight path of the projectile is determined by the future target position which is extrapolated using a simple linear trajectory model. The gun system is supposed to be a highly effective 40-millimeter gun.

After the firing of the projectile, the radar keeps on tracking the target which is supposed to undertake an evasive motion. Its future position is updated using a sequence of tangents at the real trajectory. The radar must also track the projectile in order to estimate the angular deviation required to reach the intercept point.

The angular attitude of the projectile is measured by means of the onboard magnetic sensors designed by ISL [3] and transmitted to the base station. As a matter of fact, the roll angle is needed to trigger the relevant lateral impulse thruster in the direction of the intercept point.

The flight path of the projectile is deflected using a series of nearinstantaneous lateral impulses. The effect of a simple lateral impulse has been 
investigated at the Army Research Laboratory [4]. The original feature of the concept is that the angular motion of the projectile is controlled by series of double impulses in order to sum their effect with the action of the lift force resulting from the angular motion.

The impulse thrusters are small detonators designed by ISL. They are arranged inside the projectile in the form of a ring of lateral divert thrusters. Each of them can deliver an impulse of approximately $1 \mathrm{~N} \cdot \mathrm{s}$.

\section{NAVIGATION AND CONTROL}

A two-axis magnetometer sensor is embedded in the projectile (Fig. 2) to measure the projections of the Earth magnetic field along two radials axis of the body-fixed frame.

By using these two measures $\left(H_{a}\right.$ and $\left.H_{b}\right)$ and the knowledge of the direction of the Earth magnetic field in the reference frame $\left(\vec{H}_{\text {ref }}\right)$, an EKF is designed to estimate the value of the roll angle $\varphi$ and the roll rate $\omega_{c}$. Then, the computation of the trigger time is possible depending on the roll angle estimation $\hat{\varphi}$, the target angle $\varphi_{d}$, and the roll

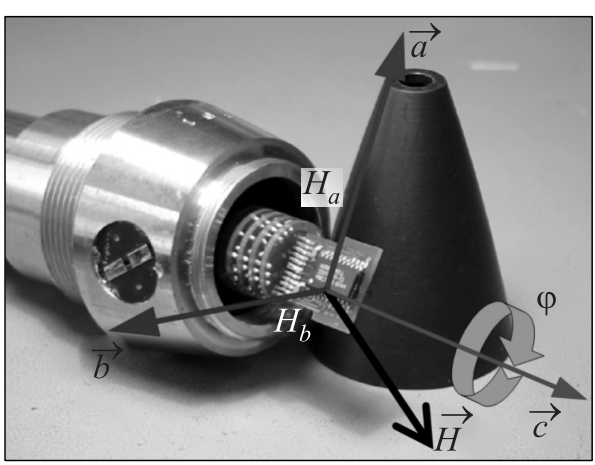

Figure 2 Two magnetometers embedded in the projectile velocity estimation $\hat{\omega}_{c}$.

\subsection{Roll Angle Estimation Using an Extended Kalman Filter}

To design the EKF to estimate the roll angle of the projectile, a dynamic modeling was developed [5].

The evolution of the projectile velocity depends on the initial velocity $v_{0}$ $\approx 1000 \mathrm{~m} / \mathrm{s}$ and on the features of the projectile symbolized by the constant $k_{0}$ :

$$
\dot{v}=-k_{0} v_{0}^{1 / 2} v^{3 / 2} .
$$

The evolution of the roll angle $\varphi$ is directly linked to the linear velocity:

$$
\omega_{c}=\dot{\varphi}=v \frac{\varepsilon}{r_{f}}
$$

where the fin cant angle $\varepsilon$ is about $0.15^{\circ}$ and $r_{f}$ denotes the distance between the axis of the projectile and the center of the fin. 
The modeling of the observation equations is a basic projection of the direction of the Earth magnetic field in the body frame. A complete model requires taking into account the trajectory and the angular motion of the projectile. Since only the estimation of the roll angle $\varphi$ is needed, some simplifications can be done. So, the following equations represent the observation model, where $H_{\text {env }}$ and $\lambda$ depend on the angular position of the projectile along its trajectory [6]:

$$
H_{a}=H_{\mathrm{env}} \cos (\lambda-\varphi) ; \quad H_{b}=H_{\mathrm{env}} \sin (\lambda-\varphi) .
$$

According to the sensors model (scale factor and offset), the following equations represent the complete observation model implemented in the EKF:

$$
Y_{\mathrm{obs}}=\left[\begin{array}{l}
L_{a} H_{\mathrm{env}} \cos (\lambda-\varphi)+R_{A} \\
L_{b} H_{\mathrm{env}} \sin (\lambda-\varphi)+R_{B}
\end{array}\right] .
$$

As the scale factor $\left(L_{A}\right.$ and $\left.L_{B}\right)$ and the offset $\left(R_{A}\right.$ and $\left.R_{B}\right)$ are unknown, it is necessary to estimate them in the EKF; so, they are included in the state vector $X$. According to the projectile's dynamics, an Euler discretization with a sample time $T_{e}=1 \mathrm{~ms}$ is sufficient. The following equations represent the evolution model implemented in the EKF:

$$
X_{k+1}=\left[\begin{array}{c}
\varphi \\
\dot{\varphi} \\
v \\
L_{a} \\
R_{A} \\
L_{b} \\
R_{B}
\end{array}\right]_{k+1}=\left[\begin{array}{c}
\varphi \\
\dot{\varphi} \\
v \\
L_{a} \\
R_{A} \\
L_{b} \\
R_{B}
\end{array}\right]_{k}+T_{e}\left[\begin{array}{c}
\dot{\varphi} \\
\ddot{\varphi} \\
\dot{v} \\
0 \\
0 \\
0 \\
0
\end{array}\right]_{k}=\left[\begin{array}{c}
X_{k}(1)+T_{e} X_{k}(2) \\
X_{k}(2)-T_{e} \varepsilon /\left(r_{f} k_{0} v_{0}^{1 / 2} X_{k}(3)^{3 / 2}\right) \\
X_{k}(3)-T_{e} k_{0} v_{0}^{1 / 2} X_{k}(3)^{3 / 2} \\
L_{A} \\
R_{A} \\
L_{B} \\
R_{B}
\end{array}\right] .
$$

The observation model can now be written as

$$
Y_{k}=\left[\begin{array}{l}
X(4) H_{\mathrm{env}} \cos (\lambda-X(1))+X(5) \\
X(6) H_{\mathrm{env}} \sin (\lambda-X(1))+X(7)
\end{array}\right] .
$$

First, the EKF is tested in a simulation. Figure 3 shows the estimation of the roll angle $\hat{\varphi}$ and Fig. 4 shows the estimation of the magnetometer measurements on the axis of the body-fixed frame.

Figure 3 presents the simulation results of the roll angle estimation. The filter algorithm starts at time $t=0.3 \mathrm{~s}$ when the roll rate has reached its steady state. Figure $3 a$ compares the reference angle with the estimation angle: one revolution seems enough to make the estimation converge. Figure $3 b$ focuses on the estimation error: after $0.1 \mathrm{~s}$, the estimation error stays under $1^{\circ}$. So, the 


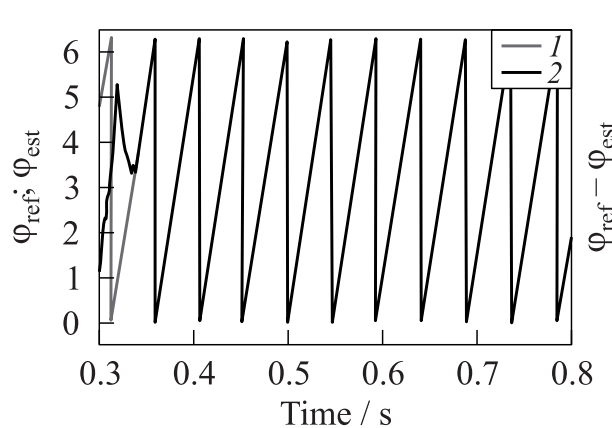

(a)

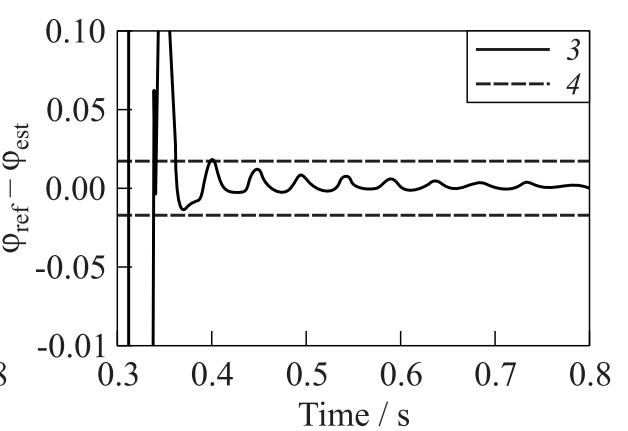

(b)

Figure 3 Roll angle estimation: $1-\phi_{\text {ref }} ; 2-\phi_{\text {est }} ; 3-\phi_{\text {ref }}-\phi_{\text {est }} ;$ and $4- \pm 1^{\circ}$

reference and estimation angles are close enough to have a good precision on the trigger estimation time.

No direct measurement of the roll angle is available on the experiment system. Thus, to test and validate the method, the magnetometer measurements and estimates are compared. The magnetometer estimates $H_{a \text { est }}$ and $H_{b \text { est }}$ are computed from the estimation state vector:

$$
\begin{aligned}
& H_{a \mathrm{est}}=\hat{X}(4) H_{\mathrm{env}} \cos (\lambda-\hat{X}(1))+\hat{X}(5) ; \\
& H_{b \mathrm{est}}=\hat{X}(6) H_{\mathrm{env}} \sin (\lambda-\hat{X}(1))+\hat{X}(7) .
\end{aligned}
$$

Figure 4 shows simulation results for the estimations of the scale factors and the offsets of the two embedded magnetometers. These four estimates converge on the simulated values. In Figs. $4 a$ and $4 b$, the estimation of the magnetometer measure $H_{a \text { est }}$ and $H_{b \text { est }}$ converges to the simulated references signals $H_{a \text { ref }}$ and $H_{b \text { ref }}$; the simulation shows that one revolution is enough to have a good accuracy.

\subsection{Trigger Time Prediction}

Considering the small size of the projectile, the computation of the algorithm on a DSP cannot be embedded. So, a high-frequency transmission between a base station and the projectile is necessary. First, sensors values are sent to the base station. Then, after the DSP computation process, the impulse trigger time is sent back to the projectile.

The impulse trigger time $t_{\mathrm{imp}}$ is predicted from the state estimated vector, the target roll angle, and the embedded clock time: 

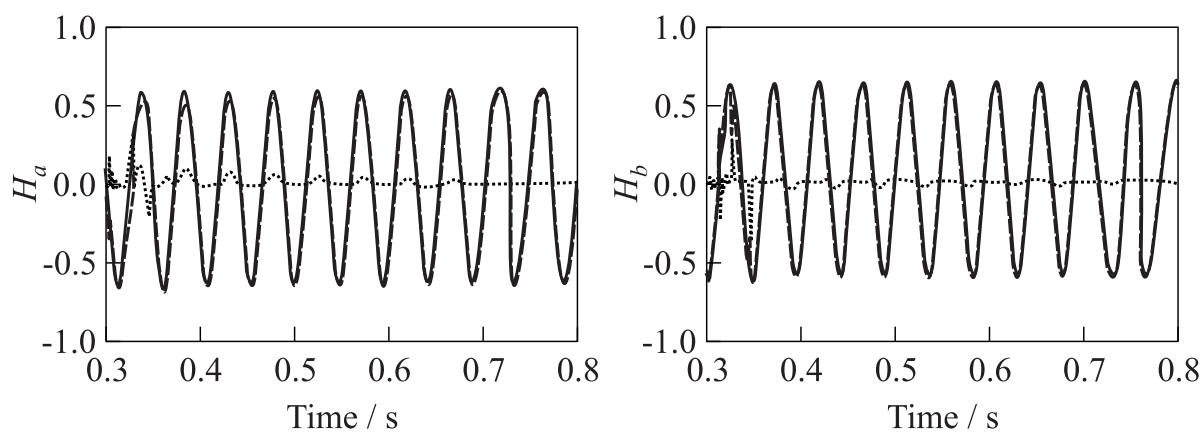

(a)

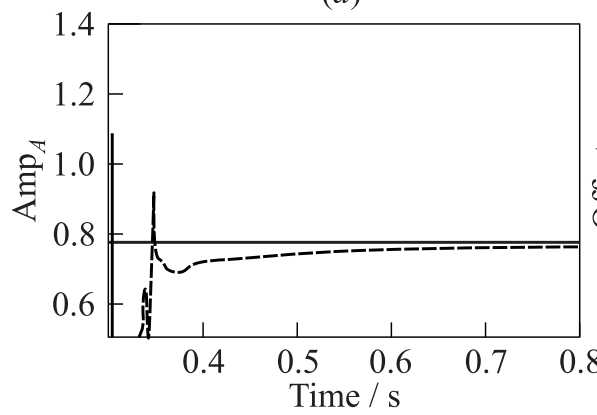

(b)

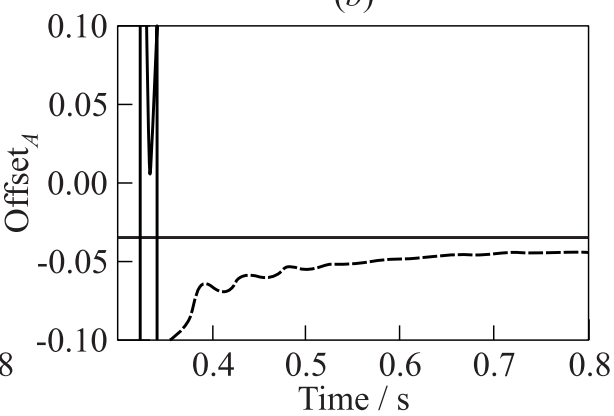

(c)
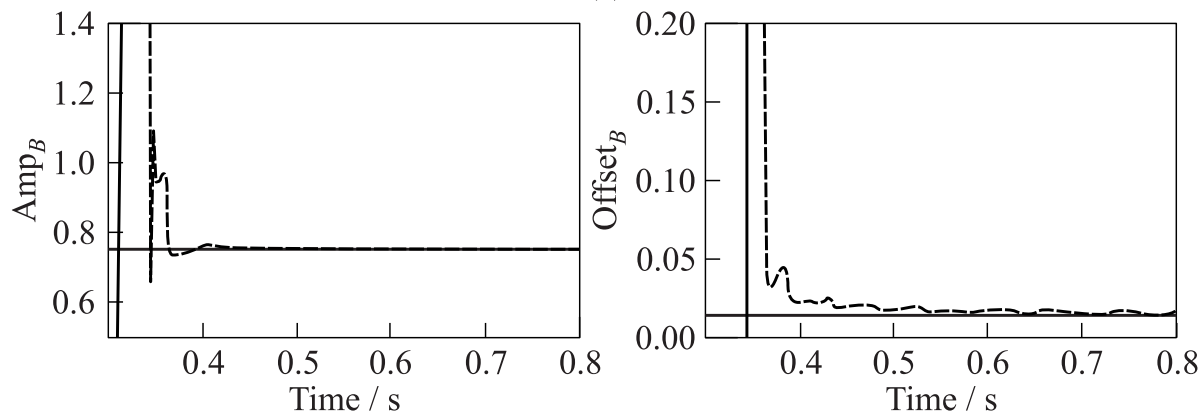

(d)

Figure 4 Magnetometers measurements estimation. Solid curves refer to reference meanings and dashed to estimated. Dotted lines show the difference $H_{a \text { ref }}-H_{a \text { est }}$ and $H_{b \text { ref }}-H_{b \text { est }}$ in $(a)$ and $(b)$, respectively

$$
t_{\mathrm{imp}}=t+\frac{\varphi_{t}-\hat{\varphi}}{\hat{\omega}_{c}} ; \quad t_{\mathrm{imp}}=t+\frac{\varphi_{t}-\hat{X}(1)}{\hat{X}(2)} .
$$

For example, Fig. 5 shows the trigger time computed for a target roll angle of $90^{\circ}$. As a new predicted trigger time is computed, the new value is sent to 


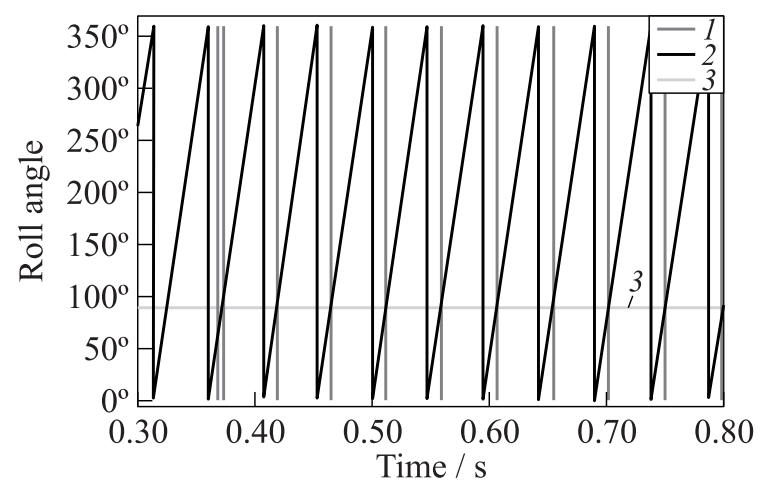

Figure 5 Predicted trigger time $(1)$ for $\varphi_{t}=90^{\circ}: 2-\phi_{\text {ref }}$; and $3-\phi_{\text {target }}$

the projectile to update the previous value. Considering the 22-hertz roll rate and the 1-millisecond sample time, the trigger time can be updated up to 40 times per revolution. The results presented on the figure show that only the first predicted trigger time is wrong, due to the delay imposed by the convergence of the algorithm.

\section{IN-LAB EXPERIMENTAL VALIDATION}

\subsection{Design of the Test Bench}

A test bench (Fig. 6) is developed according to constraints for the actuators: the motor has to reach a high-speed roll rate; the actuator should not disturb the magnet field, that is why, an electric motor cannot be used; and the motor and the electronic module have to be mounted into a safety box (Fig. 7).

It was decided to use a pneumatic motor. The choosen pneumatic motor has a maximum load speed up to 70,000 rpm (revolution per minute) under a pressure of 90 PSI ( 6 bar). The motors of this kind are disigned for grinding (with tools up to a diameter of $3 \mathrm{~mm}$ only); that is why, a lot of preliminary tests were done to make sure it is possible to spin a much larger cylinder: $38 \mathrm{~mm}$ in diameter, $90 \mathrm{~mm}$ long, with weight above $140 \mathrm{~g}$.

The motor was inserted in a polycarbonate box. This box is transparent; so, a high-speed camera can be placed in front of the LED to observe the behavior of the roll rate estimation.

The electronic module was tested with this high-speed rotation and no damages or disfunctions have been detected. 


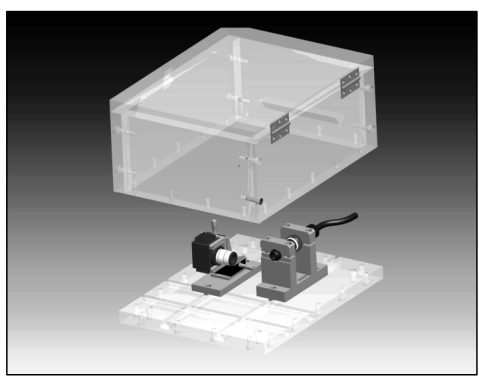

(a)

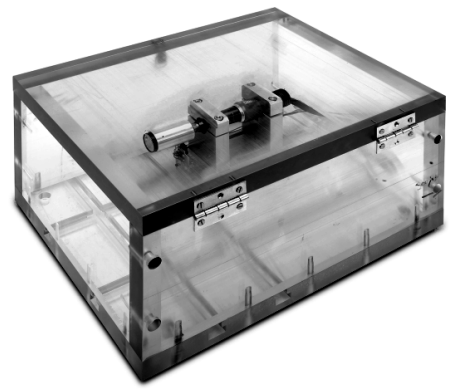

(b)

Figure 6 The CAD view of the test bench $(a)$ and the manufactured one $(b)$
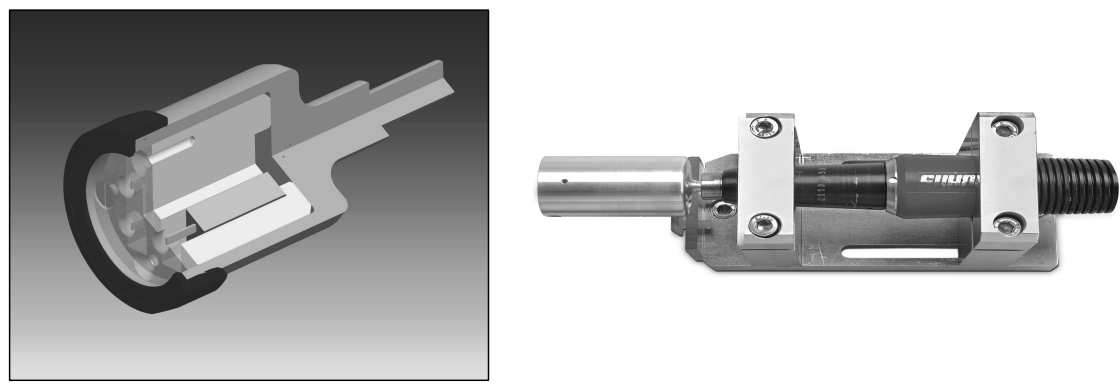

Figure 7 Shell on which the electronic module is embedded

\subsection{Experimental Results}

For the experiments, high-speed camera with an acquisition speed up to 250000 frames/s was used. In this case, the rotation frequency reached was about $950 \mathrm{~Hz}$. The images were processed to find the pictures on which the LED is switched on. A picture can be seen as a matrix with RGB levels. The white color has the highest value which is 255 . The first method could be finding the value 255 within a picture to isolate the picture with light on. This cannot work because there is noise on the pictures. The method used was to calculate the mean value of each matrix. So, the pictures on which the light is switched on correspond to the highest mean values (Fig. 8).

The mean values of the pictures on which the light is on are higher than 50 while in the other case, the mean values are between 32 and 37 (Fig. 9). A threshold value allows to extract the desired pictures.

Studying of the roll frequency shows that the value is not constant during $2 \mathrm{~s}$ of the measurement. At the beginning, the frequency is $951 \mathrm{~Hz}$ and at the end - 

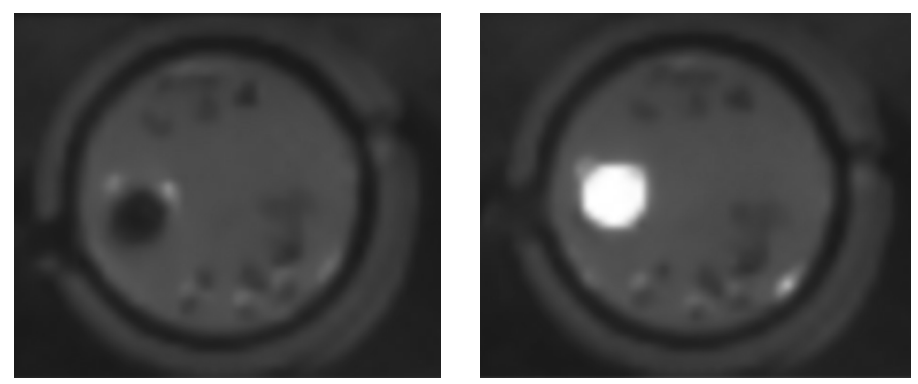

Figure 8 Pictures obtained with the high-speed camera

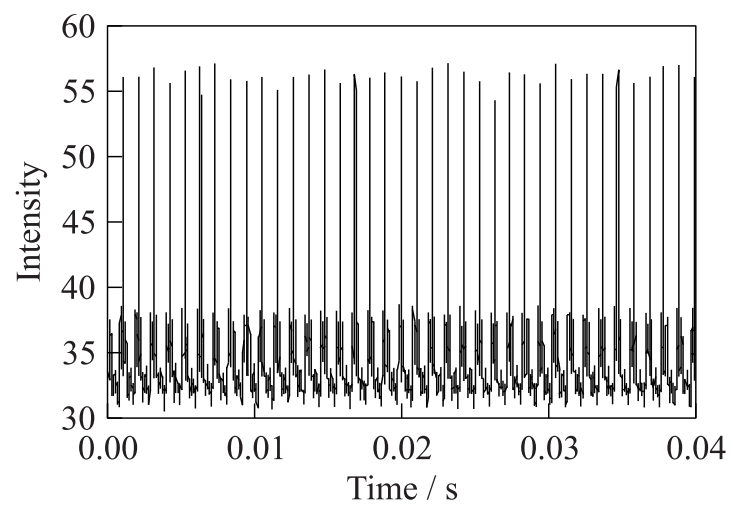

Figure 9 Mean luminous intensity of the pictures (see Fig. 8)

close to $949 \mathrm{~Hz}$. The medium value is $950.46 \mathrm{~Hz}$. This variation converted to a time spread $(2.21 \mu \mathrm{s})$ and then to the angular orientation leads to an error of $0.75^{\circ}$. At first, this error was considered negligible.

The second step was to determine the time between successive trigger events of the LED in order to quantify the jitter (Fig. 10).

First result leads to a standard deviation of up to $30 \mu \mathrm{s}$ which means, for this frequency, a precision of about $10^{\circ}$.

The Kalman filter developed was not specifically designed to be only used at a 1000-hertz roll rate. The first purpose was to scan a range between 100 and $1000 \mathrm{~Hz}$ with the same filter.

In the previous study, some experiments were made on the filter by simulating the magnetometer signals with a function generator. The maximal standard deviation obtained was less than $1^{\circ}$ over all this frequency range. That is why the accuracy of the algorithm developed is expected to be improved. 


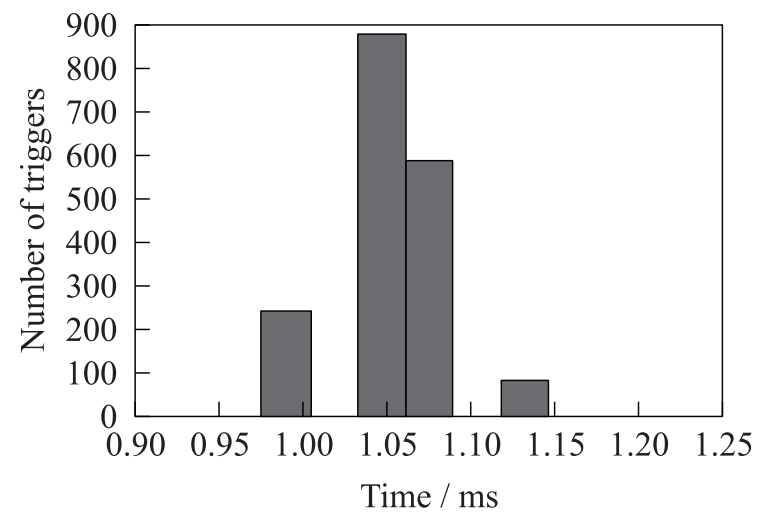

Figure 10 Distribution of the time between the trigger of the LED

\section{CONCLUDING REMARKS AND OUTLOOKS}

The hardware developed resists to the high-speed rotation. The first results have shown that the accuracy of the roll angle estimation is not as expected. Further investigations are needed to reduce the standard deviation. For that, it is decided to embed a recorder and process those data to see if the errors come from the algorithm or from the software clock that generates the trigger events.

\section{REFERENCES}

1. Changey, S., E. Pecheur, and P. Wey. 2009. Real-time estimation of supersonic projectile roll angle using magnetometers: In-lab experimental validation. 2nd IFAC Workshop on Dependable Control of Discrete Systems. Bari, Italy.

2. Wey, P., C. Berner, E. Sommer, V. Fleck, and H. Moulard. 2005. Theoretical design for a guided supersonic projectile. 22th Symposium (International) on Ballistics. Vancouver, BC, Canada.

3. Fleck, V., R. Meyer, and E. Sommer. 2004. Onboard measurements of the motion of projectiles with accelerometers and magnetometers. 21st Symposium (International) on Ballistics. Adelaide, Australia.

4. Guidos, B. J., and G.R. Cooper. 1999. The effect of a simple lateral impulse on kinetic energy projectile in flight. Report ARL-TR-2076. Army Research Laboratory.

5. Fleck, V. 1998. Introduction a la Balistique Extérieure. France: Institut FrancoAllemand de Recherche de Saint-Louis.

6. Changey, S. 2005. Modélisation et estimation par filtrage non linéaire de l'attitude d'un projectile à partir de magnétomètres. Ph.D. Thesis. Supélec, France. 